\title{
Eastern District (Wan Chai) Dispensary and Plague Hospital
}

\author{
Moira Chan-Yeung, FRCP, FRCPC \\ Member of the Education and Research Committee, Hong Kong Museum of Medical Sciences Society
}

https://doi.org/10.12809/hkmj-hkmms201912

The Eastern District (Wan Chai) Dispensary was one of the 10 Chinese Public Dispensaries (CPDs) established by the Chinese elite under the supervision of the Registrar General (later renamed Secretary for Chinese Affairs) in Hong Kong. The CPDs provided much-needed Western medical out-patient and midwifery services to the impoverished Chinese during the first half of the twentieth century and were the forerunners of the government out-patient clinics of today. The CPDs were more than conventional dispensaries. They not only popularised Western medicine but also played a crucial role in imparting public health service in Hong Kong, including free vaccination and inoculation, and education of the public in basic hygiene and sanitation.

As early as 1892, the Colonial Surgeon proposed establishing dispensaries staffed by licentiates of the Hong Kong College of Medicine to perform birth and death registration-data that Hong Kong lacked-and to provide Western medical services to the Chinese. ${ }^{1}$ The lack of funding and the outbreak of plague in 1894 overwhelmed existing resources and the project was not implemented. In the end the CPDs were founded by the Chinese elite for a different reason.

During the plague epidemic of 1894, large number of dead bodies were "dumped" in the streets. To avoid the invasive measures imposed by the draconian public health law, which required cleansing of the house and furniture and isolation of the patients with infectious diseases such as plague, household members hid those with disease from public health officials and "dumped" their bodies in the streets when they died.

This dumping of bodies in the streets was regarded by the Europeans as a gross indecency and it became such a huge scandal that two Chinese members of the Sanitary Board, Mr Wa-chun Fung and $\mathrm{Mr}$ Chu-pak Lau, decided to do something about the problem. They approached the Chairman of Tung Wah Hospital Committee to establish CPDs with a licentiate of the Hong Kong College of Medicine in attendance to identify the cause of death of dumped bodies. Two CPDs were opened on 1 April 1905 in areas with high population density: Western and Eastern District, followed by a third in Kowloon City the same year. They were managed by a committee consisting of the Registrar General, $\mathrm{Mr}$ AW Brewin, Wa-chun Fung, Chu-pak Lau, and the chairman of Tung Wah Hospital. ${ }^{2}$ The success of the first three CPDs led to the establishment of seven more in subsequent years, in Yau Ma Tei, Hung Hom, Central District, Shau Kei Wan, Sham Shui Po, Aberdeen, and Stanley.

Soon after the founding of the first three CPDs, the committee decided to operate independently from the Tung Wah Hospital. The committee, called the CPD Committee, was chaired by the Registrar General, and enlarged to include a total of 19 people from prominent members of the Chinese community. ${ }^{3}$ In 1909, Dr Ho Kai was the vice chairman of the CPD Committee.

The CPDs were usually built on land gifted by the government, with funds for construction raised locally by the Chinese elite. Operating expenses came mainly from subscriptions from the local community, the Chinese elite, and funds raised by special performances put on by local theatres. ${ }^{4}$ Over the years, the government gave small grants of $\mathrm{HK} \$ 2000$, less than $10 \%$ of the total expenditure, to the CPD Committee. ${ }^{5}$ The Registrar General controlled the funds for the CPDs on Hong Kong Island; but those in Kowloon had their own local committees responsible for their finances.

The main tasks of the CPDs included: (1) to establish the cause of death of those unattended to by a licenced doctor and to issue death certificates; (2) to inspect dead bodies at the request of the Sanitary Department or the Police to determine the cause of death, and to remove corpses to a hospital or mortuary; (3) to diagnose and treat patients who came to the dispensary and to arrange for the seriously ill to be sent to the hospital; (4) to be responsible for plague patients in the district plague hospital and to arrange for plague patients to be sent out of Hong Kong at their request; and (5) to perform smallpox vaccination and cholera inoculation. ${ }^{6}$

Soon, other duties were added. In 1906, the government hired midwives, who had been trained in Western methods of delivery, to be attached to some CPDs. Residents who wished to have their services for domiciliary delivery could apply through the medical licentiate of the CPD. ${ }^{7}$ In 1930, a gynaecology clinic was held by one of the government 
lady medical officers once a week in each of the CPDs for women with gynaecological problems. The CPD Committee also organised "lecturers" to propagate basic hygiene and public health measures in the local community and to encourage compliance to sanitary regulations. ${ }^{8}$

The Eastern District Dispensary was established in 1905 at 205 Queen's Road East, located in a ground floor shop with inadequate space. In 1907, Dr Ko-tsun Ho became the licentiate in charge of the dispensary. He encouraged the residents to campaign and raise funds for a more spacious dispensary and a small plague hospital. A door-todoor collection yielded the required amount for the purchase of a suitable location on Stone Nullah Lane (IL 1835) and for construction of the dispensary. The government granted an adjacent area of 62 feet by 40 feet (IL 790), thus expanding the site. The complex, which was opened officially on 7 October 1911 (Fig), included a small plague hospital on the top floor, a dispensary on the ground floor. ${ }^{9}$ There were also waiting rooms, quarters for coolies, and space for an ambulance and dead-vans.

In the late 1910s, there were very few plague patients. At the request of the Eastern District Dispensary Committee, the plague hospital began to admit women and children with other diseases on the understanding that the hospital should be staffed by a duly qualified medical practitioner under the supervision of the Principal Civil Medical Officer. In 1919, the plague hospital was converted into a maternity hospital with 22 beds. Dr Alice Hickling, who was responsible for training government midwives, was asked to supervise the maternity hospital with Dr Ko-tsun Ho. ${ }^{10}$ The maternity hospital became highly popular and the number of deliveries exceeded 1000 per year in 1931. At that point, altruistic residents in Wan Chai began to

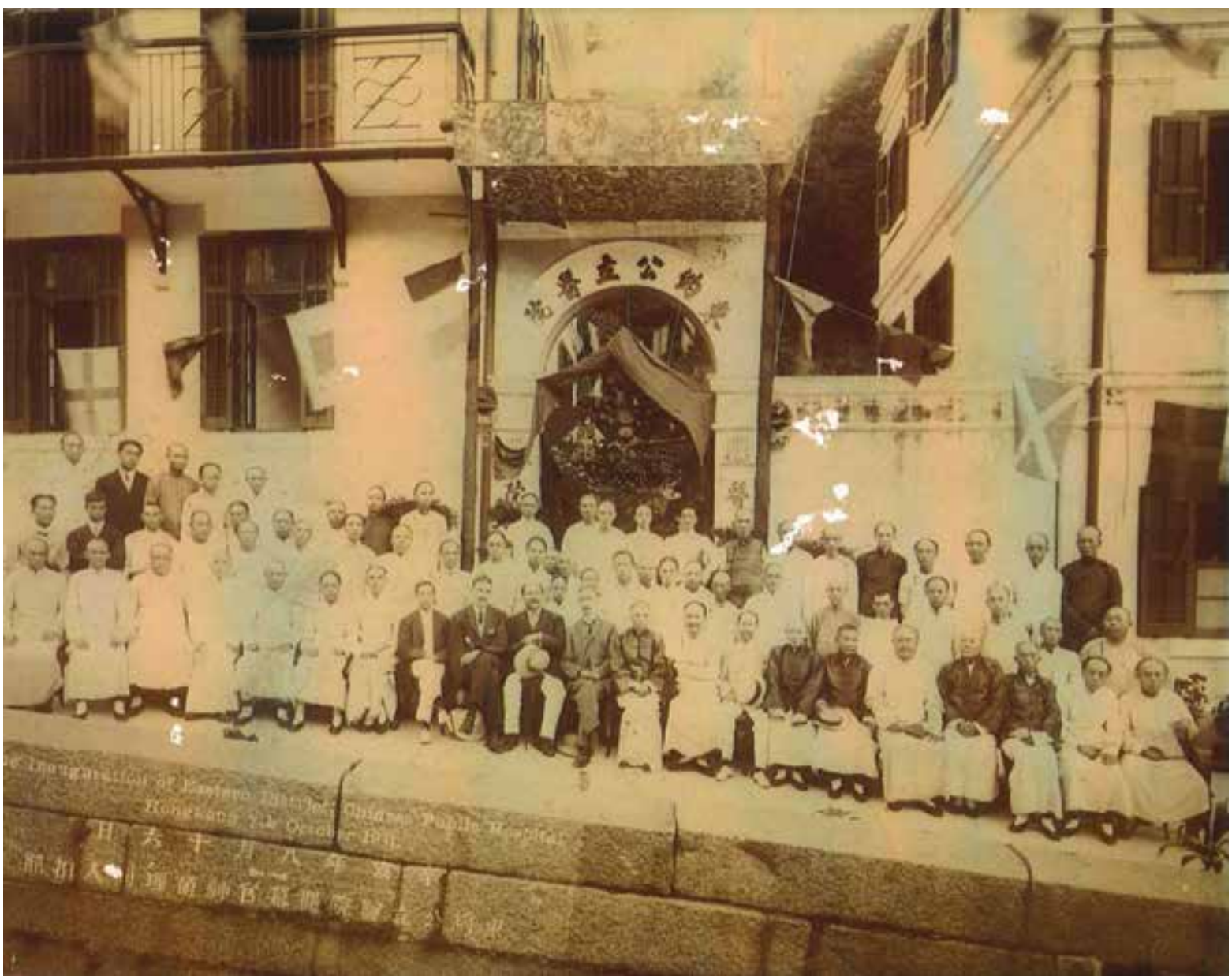

FIG. Inauguration of the Eastern District (Wan Chai) Chinese Public Dispensary and Plague Hospital at Stone Nullah Lane on 7 October 191I. On the front row were Dr Ho Kai (with a hat), Registrar General Mr AW Brewin (on his left), and Ho Ko-tsun Ho (second person to his right), members of the Chinese Public Dispensary Committee and members of the Wan Chai Kaifong Committee. The photograph was a copy of the original donated to the Hong Kong Museum of Medical Sciences by Dr Frank Cheng. One of the relatives of $\mathrm{Dr}$ Cheng, a member of Kaifong Committee, contributed and raised funds for the building of this dispensary and plague Hospital 
collect money to expand the hospital. At the end of 1931, a new maternity ward appeared on the second floor.

During the Second World War, Dr Ho used his own resources to keep the Eastern District Dispensary opened for the residents of Wan Chai. After the War, the government took over the management of the Eastern District Dispensary and Maternity Hospital. In 1949, Dr Ko-tsun Ho's meritorious work at the dispensary was recognised by an award of OBE (Order of British Empire). He had just retired from the dispensary without any pension after several decades of devoted service to the community. The Secretary for Chinese Affairs allowed Dr Ho to continue living on the second floor of the dispensary where he had lived for over 30 years without paying rent, at the petition of the residents in the neighbourhood who had great respect and admiration for the doctor. ${ }^{11}$

In 1969, all clinical activities at the dispensary were moved to the new Tang Chi Ngong Specialist Clinic next to the Tang Shiu Kin Hospital. The Eastern District Dispensary and Maternal Hospital came to an end. The premises on IL1835 and IL790 were then leased to three different organisations: the north wing to St James' Settlement, the central part to Wan Chai Kaifong Welfare Association, and the south wing to Lok Heep Club, Caritas. ${ }^{12}$ Today, the original site is occupied by the St James' Settlement Headquarters building.

\section{References}

1. Ayers to Colonial Secretary 18 August 1892, enclosed in Robinson to Lord Knutsford, 23 September 1892, CO 129/256, \#280, 207-10.

2. “Sanitary Board Fortnightly Meeting”. South China Morning Post 15 March 1907.11; Report of the Registrar General for the Year 1905; Hong Kong Sessional Papers, 13/1906, 230-1.

3. Registrar General Report 1907; Hong Kong Sessional Papers (HKSP) 1908, 191-2.

4. Secretary for Chinese Affairs (SCA) Report 1936/1937, Hong Hong Administrative Report (HKAR) 1937, C47.

5. Registrar General Report 1908. HKAR 1908, C7; “Grant for Chinese dispensaries”, South China Morning Post, 22 December 1916.

6. Registrar General Report 1910. HKAR 1910, C10, C34.

7. Registrar General Report 1907. HKSP 1908, Table IX.

8. Registrar General Report 1910. HKAR 1910, C10.

9. Registrar General Report 1911. HKAR 1911, C11-C12; IL= Inland Lot.

10. SCA Report 1920. HKAR 1920, C12, 67.

11. Ho FC. Western Medicine for Chinese. Hong Kong: Hong Kong University Press; 2018: 145.

12. SCA to Hon Colonial Secretary 30 September 1964 Chinese Public Dispensaries (Winding Up) Bill, Hong Kong Public Records Office, MD 227/50, HKRS 1394-1-26. 